\title{
La infuencia de la habitabilidad de la vivienda en los patrones de convivencia familiar
}

\author{
Víctor Corral* \\ Ivan Lohr* \\ Lorenia Torres* \\ Arturo Acuña* \\ Sheila Velardez* \\ Dora Ayala* \\ Carlos E. Peña* \\ Mariana Milán**
}

\section{Resumen}

Basándonos en antecedentes de investigación que muestran que viviendas con características de mala habitabilidad propician episodios de violencia en la pareja, así como maltrato infantil, nos propusimos estudiar los procesos opuestos a la violencia familiar y la mala habitabilidad. El presente estudio investigó la posible influencia de las condiciones de una adecuada habitabilidad de las casa en la convivencia positiva de familias mexicanas. Se procesaron las respuestas que dieron 205 habitantes de una ciudad del norte de México, a un instrumento que medía las variables de interés arriba señaladas. Las condiciones de habitabilidad medidas incluyeron el control del ruido, temperaturas agradables en la casa, ausencia de hacinamiento, iluminación apropiada, profundidad, y privacidad. Por su parte, los patrones de funcionamiento positivo familiar consideraron situaciones como el apoyo familiar, la comunicación entre los integrantes, la toma de decisiones democrática, la flexibilidad, el afecto demostrado, la aceptación y el respeto a las opiniones de todos, entre otras. Un modelo de ecuaciones estructurales reveló una correlación significativa entre la habitabilidad de la vivienda y la convivencia positiva, lo cual sugiere que las condiciones físicas de una casa pudieran constituir elementos importantes que estimulan una sana interacción entre los integrantes de la familia, así como su bienestar.

\section{Abstract}

Based upon antecedents showing that a negative housing habitability propitiates inter-partner violence as well as child maltreatment, we aimed at studying the processes that are opposite to family violence and a bad habitability. This study investigated the possible influence of adequate housing habitability on the positive functioning of Mexican families. Two-hundred-and-five inhabitants from a northern Mexican city responded to an instrument assessing the two above-mentioned variables. The assessed habitability conditions included noise control, pleasant temperature, a lack of overcrowding, appropriate illumination, depth, and privacy. In turn, positive family functioning considered situations such as family support, family members' communication, democratic decision-making, flexibility, display of affection, acceptance and respect of everybody's opinions, among others. A structural equations model revealed a significant correlation between housing habitability and positive family functioning, which suggests that the physical conditions of a house could shape important elements stimulating a healthy interaction among family members, as well as their well-being.

*Universidad de Sonora

**Universidad Nacional de San Luis, Argentina

Palabras clave: habitabilidad, vivienda, convivencia, familia. 


\section{Introducción}

El tema de habitabilidad en la vivienda y cómo ésta influye en la convivencia familiar es relativamente nuevo para los investigadores sociales; el estudio a profundidad de temas como éste surge de la necesidad de explicar por qué los diferentes factores que afectan el hábitat de un individuo pueden verse reflejados en el tipo de convivencia que las personas tienen con los demás integrantes de su familia (Holman y Stokols, 1994; Rueda, 2004). La relación entre habitabilidad y convivencia familiar se presenta como lógica debido a que el funcionamiento de la familia ocurre en un escenario socio-físico delimitado por las condiciones materiales de la vivienda, así como por las percepciones que las personas elaboran de ese escenario.

La teorización en psicología ambiental, así como el desarrollo de estudios empíricos previos, que serán comentados con mayor detenimiento en este artículo, indican que la habitabilidad de una casa puede predisponer a los integrantes de una familia a desplegar pautas comportamentales agresivas como la violencia intramarital y el maltrato infantil (Corral, Frías \& González, 2010; Corral, Barrón, Cuén \& Tapia, 2011; Holman y Stokols, 1994; Landázuri y Mercado (2004). Lo anterior señala la pertinencia de estudiar factores de habitabilidad relacionados con el funcionamiento de las familias.

En la medida en que esos factores se vean identificados sería posible generar estrategias de intervención (prevención, correctivas) que enfrenten toda la gama de problemas derivados de la convivencia familiar, especialmente la ruptura y disfunción familiar, así como la violencia en la pareja y el maltrato a los hijos (Corral et al., 2011). Desde un enfoque más positivo, el estudio de los factores que promueven una convivencia familiar sana ayudaría a establecer las condiciones que permitan un crecimiento armónico de los integrantes de la familia, y, en general, el bienestar psicológico y físico de los mismos.

\section{Habitabilidad de la vivienda}

El concepto de habitabilidad refiere las condiciones en las que una familia ocupa una vivienda. Estas condiciones están determinadas por las características físicas de la casa, la percepción que tienen las personas de estas características, así como por factores psicosociales de la familia. La habitabilidad pone de manifiesto la satisfacción que obtiene una persona de un escenario o grupos de escenarios (Mercado, 1998). Dado que la casa se constituye en el escenario de la interacción familiar, es probable que la satisfacción promovida por la habitabilidad pueda manifestarse en patrones de convivencia positiva entre los integrantes de una familia (Landázuri \& Mercado, 2004).

La habitabilidad es un constructo de orden superior, es decir, se conforma de las interrelaciones entre factores (constructos de primer orden) que denotan las condiciones físicas, sociales y los aspectos preceptuales arriba mencionados. Dentro de los factores de la habitabilidad se encuentra el hacinamiento. Éste se concibe como un estado percibido por el individuo, el cual es resultado de obtener una menor privacidad a la deseada o esperada y se relaciona con la cantidad de espacio disponible por persona en un área determinada, así como con la posibilidad de controlar el acceso a la información de uno mismo que otros pueden obtener (Hombrados, 2010). Santoyo y Anguera (1992) señalan que a finales del siglo XX el fenómeno del hacinamiento se encontraba entre los más preocupantes para los científicos sociales y representaba un problema de salud pública de gran interés. Desde el punto de vista psicológico el hacinamiento implica varios aspectos derivados de la ruptura de un equilibrio de interacción con el medioambiente, tanto como fenómeno individual; como grupal, lo que genera una serie de reacciones dirigidas a la restauración de tal equilibrio, el que implica una transacción óptima con el entorno para el logro de una serie de objetivos individuales o colectivos. 
La privacidad, por otro lado, es una dimensión adicional de la habitabilidad. Altman (1975), la define como el control selectivo del acceso a uno mismo o al grupo al que pertenece el individuo. Valera y Vidal (2010, p. 120) la conceptúan como "la capacidad de la persona o grupo de personas de regular - controlar selectivamente la cantidad e intensidad de contactos e interacciones sociales en un contexto socioambiental determinado". La privacidad en el hogar implica, entonces, contar con espacios que le permitan no sólo a la familia, sino a cada uno de sus integrantes, controlar aspectos de intimidad, la posibilidad de estar solo o disponer de condiciones para obtener tranquilidad.

También se describen dimensiones físicas de la habitabilidad como la temperatura: Una casa que amortigua el frío exterior en el invierno y el calor en el verano, es una casa con buenas condiciones de habitabilidad. La iluminación juega un rol importante, en la medida que posibilita la realización de un buen número de actividades esenciales al interior de la vivienda (cocinar, desplazarse, estudiar, etcétera). El control del ruido, tanto el proveniente del exterior como el que se genera dentro de la casa, es otra de las condiciones de la habitabilidad. Se encuentra también la profundidad, entendida esta última como el número de puertas que deben trasponerse para llegar al lugar más lejano de una casa, lo cual reflejaría el tamaño o volumen de la vivienda (Landázuri \& Mercado, 2004; Hillier y Hanson, 1988).

\section{Convivencia familiar}

La convivencia familiar, por su lado, denota la cantidad y calidad de interacciones entre los integrantes de la familia, dentro de las que pueden identificarse relaciones positivas y negativas. Algunos autores hablan de funcionamiento familiar para referirse a los patrones de convivencia entre padres, hijos, hermanos y la pareja (Noller, et al., 1992; Richardson, 1986; Zubrick, et al.,2000). Zubrick et al., (2000) resumen una serie de indicadores de funcionamiento familiar y los dividen en seis aspectos: tiempo, recursos materiales, capital humano, capital psicológico, capital social y variables confusoras.

Los indicadores de tiempo incluyen el empleo (de tiempo completo o parcial) materno y paterno, las horas de tiempo pagado y no pagado. Comprende además las actividades e interacciones de los padres con los hijos: tiempo total de interacción, actividades entre padres e hijos, comidas juntos, salidas, actividades escolares, lectura a los hijos, ayuda en tareas, tiempo en actividades recreativas, tiempo viendo juntos TV o videojuegos o internet, tiempo con el(la) esposo(a), tiempo en trabajo casero. Los recursos materiales consideran el ingreso total, las fuentes del ingreso, las posesiones familiares, relación posesionesdeudas, grado de pobreza, cobertura de salud, status de posesión de la vivienda (propia o de renta).

El capital social incluye la educación de los padres, su estado de salud física; también prácticas de crianza como técnicas disciplinarias, reglas de conducta y en la casa, monitoreo de hijos, comunicación padreshijos, frecuencia y estilos de comunicación, paternidad consistente, interacción positiva, aceptación y castigo, paternidad aversiva, modelamiento, conocimiento culturalmente adquirido, creencias, actitudes, valores y tradiciones. El capital psicológico, a su vez, está constituido por la cohesión familiar, la relación entre integrantes, la salud mental de los padres, la satisfacción con la paternidad y el empleo, el estrés percibido, la auto-eficacia de la paternidad, el hacinamiento, el número de hijos, y la movilidad residencial. El capital social tiene que ver con la confianza, el involucramiento cívico, la reciprocidad, la participación en la comunidad, las conexiones en el barrio, contactos con familia y amigos, tolerancia a 
la diversidad, disponibilidad de servicios de apoyo y la filantropía, entre otras. Por último, entre los factores de confusión mencionan la paternidad biológica vs la adopción, el status marital (soltero, casado, padre solo), costos del cuidado infantil, etcétera.

Aunque en su esquema, Zubrick et al., (2000) enfatizan las relaciones entre los miembros de la pareja y entre ellos y los hijos; el funcionamiento familiar considera también las interacciones entre hermanos y entre otros miembros de la familia (Noller et al., 1992). De acuerdo con este esquema ampliado, la convivencia familiar puede ser afectada por diversos factores. Algunas investigaciones han relacionado la estructura familiar (tamaño de la familia, el espaciamiento entre hermanos, y el sexo) y los efectos de la cantidad de tiempo que los adolescentes pasan con los padres como una medida de la calidad de la relación, y así poder establecer una de las medidas de disciplina. Richardson, et al. (1986) vincularon las variables estructura familiar con la cantidad de tiempo que los adolescentes pasan con sus familiares y la calidad de ese tiempo. Montemayor (1982) encuentra que la relación es compleja entre la cantidad de tiempo que dedican los adolescentes para estar con sus familiares y compañeros, y la influencia de la familia en el funcionamiento de la variable de conflicto. Piaget (1969) y Harner (1982) sugieren que es conveniente preguntar a los adolescentes, después de los 11 años, su percepción de la calidad y la duración del tiempo en compañía de los demás.

Los aspectos económicos son de importancia en el funcionamiento familiar en la medida en la que un bajo salario de los padres puede inducir frustración y estrés; y esto, a su vez, se puede manifestar en patrones de convivencia negativa, como la violencia (Zubrick et al., 2000). Lo económico también influye en la habitabilidad de la casa, ya que se requiere de un ingreso suficiente para construir un hogar con condiciones físicas apropiadas y garantizar su mantenimiento. Sin embargo, centrar el desarrollo familiar en el ingreso económico puede ser contraproducente. Los niños pueden vivir en familias ricas en ingreso, pero pobres en la calidad de tiempo que los padres tienen disponibles para sus hijos, dado que su esfuerzo está centrado en la acumulación de bienes materiales (Zubrick et al., op. cit.).

Sin hacer a un lado los aspectos arriba referidos, Noller et al. (1992) enfatizan el apoyo familiar, la comunicación entre los integrantes, la toma de decisiones democráticas, la flexibilidad, el afecto demostrado, la aceptación y el respeto a las opiniones de todos, como situaciones a evaluar dentro del funcionamiento familiar. De acuerdo con su esquema, una familia exhibe un funcionamiento positivo si sus integrantes se ayudan y protegen; si existe entre ellos una comunicación fluida y sincera; si en la familia se manifiesta una participación de todos en los procesos de toma de decisiones, especialmente las trascendentales; si se evita la rigidez en el trato y en las tomas de decisiones (sobre todo de parte de los padres), si se producen muestras de cariño hacia los hijos, padres, hermanos y la pareja; y si se respetan y toman en cuenta las opiniones de todos, independientemente de la edad, el género o el grado de responsabilidad dentro del grupo familiar. En este tenor, Amar (1994) refiere que la familia se puede entender como una institución de integración, cooperación, e interdependencia, unida por el afecto mutuo entre sus miembros, con la finalidad última de asegurar el bien de la sociedad.

Es interesante notar que el único factor de habitabilidad de la vivienda, mencionado explícitamente como factor que puede incidir en el funcionamiento familiar, es el hacinamiento (Zubrick et al., 2000), aunque, de manera indirecta, los aspectos económicos -que determinan grandemente las condiciones de habitabilidad- también son mencionados.

Ruiz, Ropero, Amar y Amaris (2003), en el artículo "Familia con violencia conyugal y su relación 
con la formación del auto concepto", consideran a la familia como el primer núcleo protector del niño, siendo ésta la responsable de poder garantizar las condiciones para que éste logre un desarrollo físico y psicológico en armonía con el medio en el que habita. No obstante, también debe considerarse el hecho de que dentro de la familia se presentan escenarios donde se aprende el mayor número de conductas que afectan directa o indirectamente a los niños. Por ello, es importante estudiar variables tales como la violencia al interior de la familia, cómo se da la formación del auto concepto de los niños al interior de ésta y de qué manera el escenario físico y social del hogar influyen en la convivencia familiar y el bienestar de sus integrantes.

\section{Habitabilidad de la vivienda y convivencia familiar}

Levi y Anderson (1980) señalan que un alto nivel de vida objetivo (resultante ya sea de los recursos económicos, el hábitat, el nivel asistencial o el tiempo libre), puede ir acompañado de un alto índice de satisfacción individual, bienestar o calidad de vida. Las condiciones de habitabilidad en que un individuo vive son partes del nivel de vida objetivo e influyen, por lo tanto, en su satisfacción personal y bienestar físico. De manera más específica, Rueda (2004), haciendo referencia a la convivencia familiar positiva, menciona que no tiene nada de extraño que el objetivo de la sostenibilidad social se haya enfocado en la habitabilidad, es decir, en la pretensión de mantener la calidad en la convivencia de los individuos que habitan los sistemas urbanos.

Desde los años 90 del siglo pasado y principios del presente siglo, autores como Holman y Stokols (1994) y Landázuri y Mercado (2004) habían especulado acerca de la posibilidad de que las malas condiciones de habitabilidad de una casa podrían potencialmente ser disruptores de los patrones de convivencia familiar; el planteamiento de estos autores establecía la posibilidad de que la mala habitabilidad indujera violencia entre los integrantes de la familia. No obstante, hasta apenas muy recientemente esta especulación se corroboró empíricamente.

Los únicos estudios, hasta donde sabemos, que han abordado la relación entre habitabilidad de la vivienda y la violencia familiar fueron desarrollados por Corral et al., (2010) y Corral et al.,(2011). En el primer estudio los autores reportan una asociación significativa entre la habitabilidad y la violencia, concluyendo que las condiciones negativas de habitabilidad (alta o baja temperatura, hacinamiento, poca profundidad, ruido y poca privacidad) predicen directamente la violencia en la pareja y el maltrato hacia los hijos. Los autores, basándose en la propuesta de Herrenkohl y Herrenkohl (2007) -quienes conciben los factores negativos físicos y de diseño de la vivienda que incitan la violencia familiar como "estresores familiares"sugieren que esta relación podría ser mediada por el estrés, el que sería inducido por las malas condiciones de habitabilidad. Por lo tanto, en su estudio subsecuente, Corral et al., (2011) prueban la idea de que los factores negativos de habitabilidad promueven estrés en primera instancia, el cual sería seguido por la conducta agresiva y violenta entre miembros de la familia. Esa idea fue corroborada por los datos de su investigación empírica.

Las investigaciones arriba descritas asumen un enfoque negativo en el estudio de la relación entre habitabilidad y convivencia familiar. Es decir, se interesan en el efecto negativo (violencia) que tienen las condiciones negativas de la habitabilidad. Aunque la lógica sugeriría que las buenas condiciones de habitabilidad influirían positivamente en patrones de convivencia adecuados, es necesario probar empíricamente que vivir en una casa con buenas condiciones de habitabilidad se relaciona con patrones positivos de convivencia en la familia, tal y como lo sugiere Rueda (2004). El propósito del presente estudio es probar esa hipótesis de trabajo. 


\section{Método}

\section{Participantes}

Los individuos estudiados fueron 205 personas de la ciudad de Hermosillo, Sonora, una ciudad de tamaño medio $(700,000$ habitantes) en el noroeste de México. Se seleccionó un representante de cada familia a partir de tres sub-muestras estratificadas, siendo de clase baja el $52.2 \%$, de clase media el $39 \%$ y de clase alta el $8.8 \%$. Los participantes fueron $49 \%$ mujeres y $51 \%$ hombres. Su edad promedio fue de 32.4 años, reportando una media de 12.2 años de escolaridad. Un 31.2\% fueron amas de casa, $31.7 \%$ padres de familia y $36.1 \%$ ocupaban el puesto de hijos en el hogar, de los cuales $18.6 \%$ eran hombres y $17.6 \%$ mujeres. El ingreso mensual familiar de la muestra fue, para el $7.8 \%$, de entre 0 y $\$$ 2,500 pesos mexicanos (12.50 pesos por dólar norteamericano); para el $37.1 \%$ fue de entre $\$ 2,501$ y $\$ 5,000$; para el $17.1 \%$ resultó entre $\$ 5,001$ y $\$ 10,000$; para el $18.5 \%$ fue de entre $\$ 10,001$ y $\$ 20,000$; para el $10.2 \%$ estuvo entre $\$ 20,001$ y $\$ 40,000$; y, finalmente, para el $5.4 \%$ fue de más de $\$ 40,000$. La gran dispersión y sesgo del ingreso indican que la mayoría de las personas pertenecían a la clase baja, un rasgo representativo de la población mexicana.

\section{Instrumentos}

Se utilizaron dos escalas, la de funcionamiento familiar y la de habitabilidad de la vivienda. La primera fue traducida al español a partir del instrumento original The Family Functioning Scale (Noller et al., 1992). Esta escala consta de una serie de reactivos en formato likert de siete opciones de respuesta. Los participantes responden al instrumento señalando si están "totalmente en desacuerdo" $(=0)$, hasta "totalmente de acuerdo" $(=6)$ a reactivos que registraban situaciones de apoyo familiar, la comunicación entre los integrantes, la toma de decisiones democrática, la flexibilidad, el afecto demostrado, la aceptación y el respeto a las opiniones de todos, entre otras; todas ellas referidas a sus patrones de convivencia con su familia. Aunque la escala original contiene, tanto reactivos positivos (buen funcionamiento familiar), como negativos (funcionamiento familiar negativo); para los propósitos del presente estudio sólo se analizaron los reactivos positivos.

La escala de habitabilidad es el instrumento que utilizaron Corral et al., (2010, 2011), el que, a su vez, fue adaptado del que crearon Landázuri y Mercado (2004). La escala consta de 31 reactivos tipo likert, de los cuales 9 miden hacinamiento, 11 privacidad, 4 ruido exterior, 5 ruido interior, 1 iluminación, 2 temperatura y 2 profundidad. Las respuestas van en un continuo de fácil-difícil, suficiente-insuficiente, todo-nada, imposible-posible, siempre-nunca, adecuadainadecuada, mucho-poco, no se escucha-se escucha, mala-buena, muy caliente-fresca y muy fría-cálida (en una escala de posibles respuestas que se califican desde el 1 hasta el $6)$, como calificativos de las condiciones de su vivienda.

Se incluyó, en los instrumentos aplicados, un apartado de variables demográficas donde se indaga la edad de los participantes entrevistados, su posición en la familia, el número de hijos, el sexo, el ingreso familiar mensual, los años de escolaridad, los años que tenía la casa de construida y si la misma era propia o rentada.

\section{Procedimiento}

Las entrevistas se llevaron a cabo en diversas colonias representativas de las clases sociales que conforman la ciudad de Hermosillo. Se solicitó a los entrevistados su colaboración informada para participar en el estudio. Así mismo, se les planteó que sus datos formarían parte de una investigación y que las respuestas serían absolutamente confidenciales. 


\section{Análisis de datos}

Una vez recogidos los datos, éstos fueron capturados y procesados en el programa SPSS versión 15 para obtener estadísticas univariadas de las escalas y sus reactivos, además de los indicadores de consistencia interna (alfas de Cronbach) para las dimensiones estudiadas. Con el fin de elucidar la hipótesis central del estudio se especificó y probó un modelo de ecuaciones estructurales en el que, en primera instancia, se conformó un factor de habitabilidad de la vivienda, a partir de las interrelaciones entre los indicadores de iluminación adecuada, frescura en el verano, calidez en el invierno, ausencia de hacinamiento, ausencia de ruido, privacidad, y profundidad. La convivencia familiar se especificó en el modelo como un índice, resultante de promediar los reactivos de la escala correspondiente. También se incluyó la variable de antigüedad de la casa, como un control de las condiciones de habitabilidad. El modelo planteaba que las malas condiciones de habitabilidades serían una función de la antigüedad de la casa y, a su vez, las primeras afectarían negativamente los patrones de convivencia familiar positiva. La prueba del modelo implicó obtener los coeficientes estructurales (relaciones entre indicadores y el factor de habitabilidad, así como el de las relaciones entre este factor, la antigüedad de la casa y el índice de convivencia); también implicó el cálculo de los indicadores de bondad de ajuste. Estos indicadores señalan la correspondencia entre el modelo teórico de relaciones entre las variables especificadas y el modelo obtenido a partir de los datos (Bentler, 2006).

\section{Resultados}

La tabla 1 exhibe las medias, desviaciones estándar, valores máximos y mínimos, y coeficiente de consistencia interna (alfa de Cronbach) de las escalas utilizadas en el estudio. Para la escala de convivencia familiar positiva las personas reportaron una media general de 4.70, en un rango de posibles respuestas del 0 al 6 . Las medias para los reactivos fueron similares, como lo muestra la diferencia entre el reactivo que obtuvo el mayor puntaje ("Nuestra familia se apoya", media=5.03) y el de menor puntuación ("Los hijos participan en la toma de decisiones de la familia", media=4.43). La consistencia interna para la escala de convivencia positiva fue de alfa $=.87$.

Considerando un rango de respuestas que iba del 1 al 6, la media general para la escala de habitabilidad fue de 3.80. Ésta se obtuvo, al considerar todos los componentes del instrumento que medían ese factor, exceptuando el de profundidad (dado que fue calificado en una escala diferente) y revirtiendo los puntajes para los indicadores negativos de la habitabilidad (ruido, hacinamiento, calor, frío). Al solicitarles a los participantes que reportaran la sensación de hacinamiento dentro de sus hogares, en promedio expresaron un puntaje de 1.85; la privacidad reportada tuvo una media de 2.45 y el promedio de ruido en las casas fue de 2.48. La calificación otorgada a la iluminación fue 4.30; el calor percibido en el verano recibió una calificación de 2.90 y el frío en el invierno una de 2.86. En cuanto a la profundidad de las casas, sus indicadores revelaron un promedio de 1.59 metros desde la puerta a la recámara y 6.85 metros de distancia desde la entrada de la vivienda al punto más lejano de la casa. La subescala de hacinamiento produjo un alfa de .86, la de privacidad fue $=.75$, y la de ruido alcanzó un valor de alfa $=.73$. 


\section{Tabla 1}

Estadísticas univariadas y consistencia interna de las escalas de funcionamiento familiar y habitabilidad

\begin{tabular}{|c|c|c|c|c|c|}
\hline Escala/Reactivos & Media & DE & Min & Max & Alfa de Cronbach \\
\hline Convivencia Positiva & 4.70 & & & & .87 \\
\hline Nuestra familia se apoya & 5.03 & 1.23 & 1 & 6 & \\
\hline Todos opinan en decisiones & 4.79 & 1.25 & 0 & 6 & \\
\hline Honestidad & 4.57 & 1.47 & 0 & 6 & \\
\hline Padres de acuerdo & 4.72 & 1.39 & 0 & 6 & \\
\hline Flexibles en actividades & 4.45 & 1.56 & 0 & 6 & \\
\hline Cercanía & 4.63 & 1.41 & 0 & 6 & \\
\hline Hijos opinan reglas & 4.54 & 1.46 & 0 & 6 & \\
\hline Afecto & 4.53 & 1.41 & 0 & 6 & \\
\hline Solución conjunta de problemas & 4.95 & 1.59 & 0 & 6 & \\
\hline Animarse a tomar decisiones & 4.76 & 1.43 & 0 & 6 & \\
\hline Mostrar sentimientos reales & 4.53 & 1.51 & 0 & 6 & \\
\hline Aceptación & 4.94 & 1.34 & 0 & 6 & \\
\hline Hijos participan decisiones & 4.43 & 1.54 & 0 & 6 & \\
\hline Respeto de opinión & 4.64 & 1.40 & 0 & 6 & \\
\hline Emociones firmes & 4.75 & 1.23 & 1 & 6 & \\
\hline Solución de problemas hablando & 4.76 & 1.35 & 1 & 6 & \\
\hline Comparten intereses pasatiempos & 4.52 & 1.32 & 1 & 6 & \\
\hline Todos opinan en asuntos familiares & 4.85 & 1.18 & 0 & 6 & \\
\hline Amor aun en desacuerdo & 4.73 & 1.39 & 0 & 6 & \\
\hline \multicolumn{6}{|l|}{ Padres/Hijos hablan en toma } \\
\hline de decisiones & 4.79 & 1.34 & 0 & 6 & \\
\hline Hacinamiento & 1.85 & & & & .86 \\
\hline Desplazamiento & 1.85 & 1.22 & 1 & 5 & \\
\hline Área disponible & 2.06 & 1.30 & 1 & 5 & \\
\hline Estorbos & 2.08 & 1.24 & 0 & 6 & \\
\hline Cupo pasillos & 2.24 & 1.38 & 0 & 5 & \\
\hline Espacio entrada & 2.00 & 1.31 & 1 & 6 & \\
\hline Acceso Sala-Baño & 1.63 & 1.05 & 1 & 5 & \\
\hline Acceso Comedor-Cocina & 1.62 & 1.06 & 1 & 5 & \\
\hline Acceso Refrigerador -Lavatrastes & 1.69 & 1.21 & 1 & 6 & \\
\hline Acceso Recámaras-Baño & 1.55 & 0.99 & 0 & 5 & \\
\hline
\end{tabular}




\begin{tabular}{|c|c|c|c|c|c|}
\hline Escala/Reactivos & Media & DE & Min & Max & Alfa de Cronbach \\
\hline Privacidad & 2.45 & & & & .75 \\
\hline Silencio & 2.47 & 1.48 & 1 & 6 & \\
\hline No molestia & 2.45 & 1.35 & 1 & 5 & \\
\hline No vecinos enterados & 2.09 & 1.24 & 1 & 5 & \\
\hline Control paso de extraños & 2.33 & 1.52 & 0 & 5 & \\
\hline Privacidad & 1.93 & 1.16 & 0 & 5 & \\
\hline Miembros descanso/visita & 2.63 & 1.54 & 1 & 5 & \\
\hline Privacidad recámara & 3.13 & 1.41 & 0 & 5 & \\
\hline Baño Invisible & 2.75 & 1.53 & 1 & 5 & \\
\hline Baño insonoro & 2.19 & 1.24 & 1 & 5 & \\
\hline Cerrar ventanas insonoro & 2.60 & 1.51 & 0 & 5 & \\
\hline Cerrar persianas invisible & 2.85 & 1.59 & 0 & 5 & \\
\hline Radio/Tele Vecinos & 2.01 & 1.31 & 0 & 5 & \\
\hline Ruido & 2.48 & & & & .73 \\
\hline Ventanas ruido exterior & 2.70 & 1.96 & 0 & 5 & \\
\hline Voz baja & 1.74 & 1.18 & 0 & 5 & \\
\hline Voces vecinos & 2.02 & 1.30 & 0 & 5 & \\
\hline Ruido hogar & 3.60 & 1.29 & 1 & 5 & \\
\hline Interrumpen ruidos & 2.36 & 1.21 & 0 & 5 & \\
\hline Iluminación & 4.30 & 0.96 & 1 & 5 & \\
\hline Verano-Calor & 2.90 & 1.44 & 1 & 5 & \\
\hline Invierno-Frío & 2.86 & 1.10 & 0 & 6 & \\
\hline Profundidad & 4.22 & & & & \\
\hline Puertas a recámara & 1.59 & 1.10 & 0 & 6 & \\
\hline Metros a punto más lejano & 6.85 & 9.49 & 0 & 100 & \\
\hline
\end{tabular}


La figura 1 contiene los resultados del modelo de ecuaciones estructurales de las variables analizadas. Para iniciar, se presentan los resultados del modelo de medición, es decir, el análisis factorial que permitió conformar el constructo de Habitabilidad de la vivienda (representado por el óvalo), el cual se construyó a partir de los indicadores (representado por los rectángulos) iluminación, casa fresca en verano, casa caliente en invierno, no hacinamiento, privacidad, profundidad, no ruido y años de la casa. Todos los pesos factoriales fueron salientes y significativos $(p<.05)$ indicando que la medición de esa variable latente posee validez de constructo convergente.

El modelo estructural está representado por la relación entre el factor "habitabilidad", la variable manifiesta de antigüedad de la casa, y el índice de "convivencia familiar positiva". Esta relación se denota con flechas que ligan al factor, por un lado, con la antigüedad de la vivienda y; por el otro, con la convivencia familiar positiva. De acuerdo con el modelo, la antigüedad de la casa tiene un efecto negativo en la habitabilidad, y ésta se relacionó positivamente con los patrones de convivencia familiar. La $R^{2}$ (porcentaje de varianza explicada) para la variable dependiente "convivencia familiar" fue de .25 .

Los indicadores de bondad de ajuste del modelo revelan su pertinencia. Aunque la chi cuadrada fue significativa $(p=.014)$, los indicadores prácticos igualan o superan el punto de quiebre (.90) establecido para la bondad de ajuste (Bentler, 2006) y el RMSEA es igual a .06. Esto indica que los datos respaldan al modelo especificado.

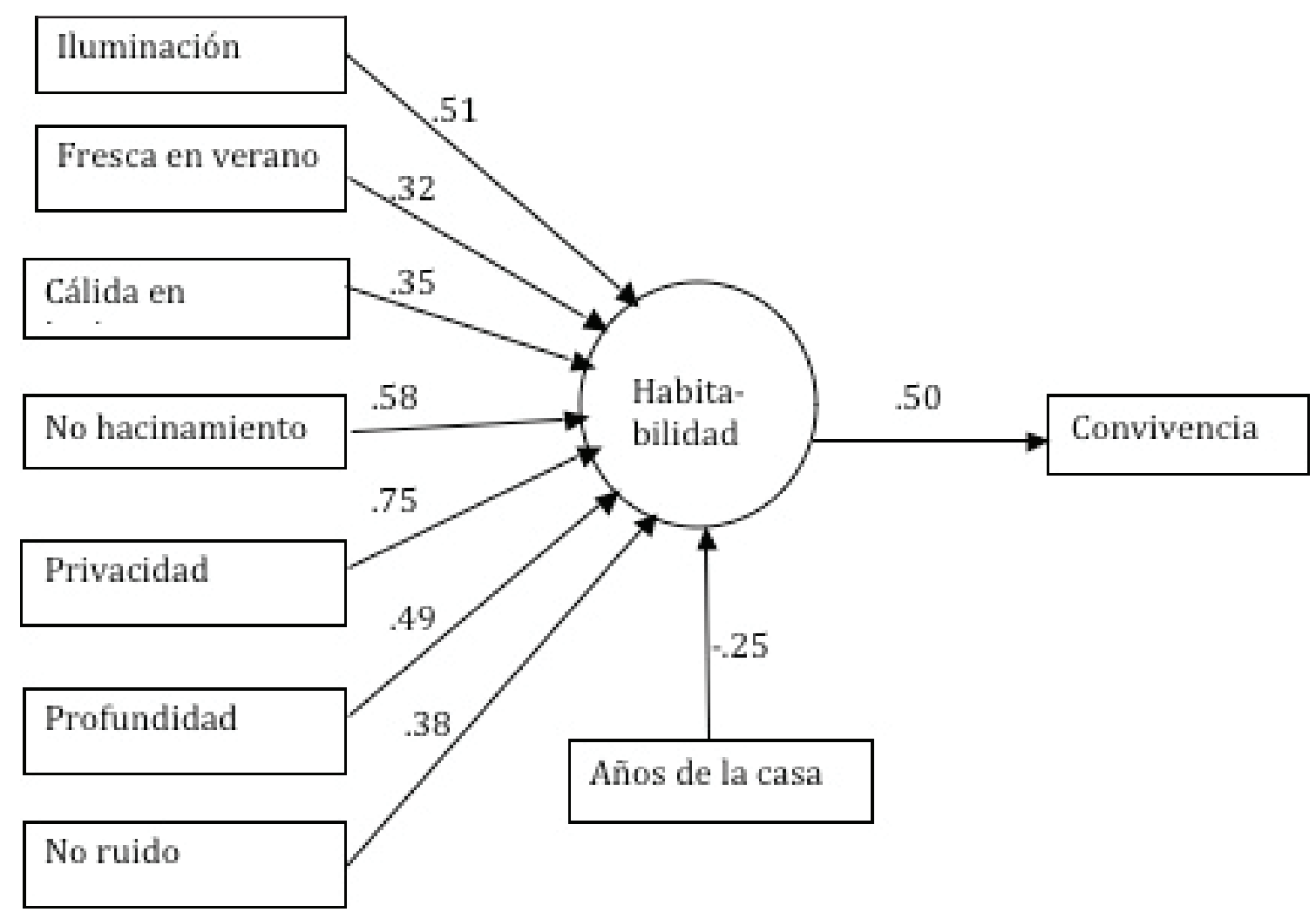

Figura 1. Modelo de la habitabilidad de la vivienda como predictor de la convivencia familiar positiva. Todos los pesos factoriales y coeficientes estructurales son significativos a $p<.05$. Bondad de ajuste: $X^{2}=41.5$ (24 gl), $p=.014 ; B N N F I=.90 ; C F I=.93 ; R M S E A=.06$. $R^{2}$ del modelo $=.25$. 


\section{Discusión}

Al igual que en los estudios previos de Corral et al., (2010; 2011), en la presente investigación la habitabilidad de la vivienda demostró afectar los patrones de convivencia familiar. Si en esos estudios las malas condiciones de habitabilidad afectaron de manera nociva el funcionamiento armónico -lo que se manifestó en estrés y en violencia intrafamiliar; en el presente estudio, los buenos niveles de habitabilidad se relacionaron con la convivencia familiar positiva. Como se esperaba, la antigüedad de la vivienda influyó en su grado de habitabilidad: las casas más viejas eran menos habitables, lo que podría explicarse por el deterioro acumulado en el tiempo y quizá la falta de mantenimiento, también acumulada.

Para medir las variables de interés aplicamos instrumentos previamente utilizados en estudios de funcionamiento familiar (Noller et al., 2002) y de habitabilidad de la vivienda (Corral et al., 2010; Landázuri y Mercado, 2004). Al igual que en las investigaciones previas, esos instrumentos arrojaron evidencias de una adecuada consistencia interna y, para el caso de la escala de habitabilidad, el modelo de medición (análisis factorial confirmatorio) de las ecuaciones estructurales sugirió validez convergente de constructo del instrumento, dada por pesos factoriales altos y significativos $(p<.05)$ de los indicadores en el factor medido. El modelo estructural reveló también que los datos respaldan la idea de que el funcionamiento familiar se relaciona con la habitabilidad de la vivienda, dado que los indicadores de bondad de ajuste práctica (NNFI y $C F I$ ) rebasan el punto de quiebre (>.90) establecido y el RMSEA es igual a .06 (ver Bentler, 2006).

Los entrevistados reportaron niveles elevados de funcionamiento familiar positivo (media de 4.7 en un rango de respuestas del 0 al 6), lo que indica que, al menos para la muestra estudiada, sus patrones de convivencia reflejaban más armonía que disfunción, manifestándose en buenas dosis de soporte que se brindaban los integrantes de la familia, sus demostraciones de afecto, la comunicación entre los miembros, la toma de decisiones compartidas, la aceptación y la honestidad entre ellos. La situación de convivencia positiva más pronunciada fue la del apoyo que se otorgan los miembros de la familia, mientras que la menos considerada fue la que refería la participación de los hijos en las tomas de decisiones. Esto podría indicar una gran preocupación por el bienestar y la seguridad de los demás (hijos, hermanos, padres, pareja), pero un aprecio no tan considerable por los estilos democráticos de conducción de las familias (más centrados en los padres), que caracterizan a las sociedades tradicionales como la mexicana.

En cuanto a las condiciones de habitabilidad, y considerando un rasgo de respuesta que iba del 1 al 6 , los participantes declararon bajos niveles de hacinamiento (1.8 en promedio) y no tan bajos niveles de calor percibido en el verano (2.9) y frío en el invierno (2.8). La privacidad expresada tampoco fue muy elevada (2.4) lo mismo que la profundidad de la vivienda (6.8 mts. desde la entrada hasta el punto más alejado de la casa); otros indicadores de habitabilidad que produjeron puntajes de alto nivel fueron la iluminación (media de 4.3) y la ausencia de ruido (el ruido fue calificado en promedio como de 2.4). Expresado de otra manera, lo anterior señalaría que, para la mayor parte de las personas entrevistadas, su vivienda, a pesar de no ser grande, no les producía una sensación de hacinamiento, aunque sí se quejaban de una relativamente pobre privacidad. Dado que el hacinamiento es un fenómeno perceptual que obedece no sólo a la densidad de personas por volumen de espacio, sino a otros aspectos de satisfacción con la vivienda y las relaciones con otras personas (Hombrados, 2010); es muy probable que, a pesar de la alta densidad y la falta de privacidad, las altas condiciones de convivencia positiva familiar estuvieran afectando la sensación de hacinamiento, de manera que las personas no lo experimentaban en niveles elevados. 
Los participantes también reportaron condiciones de poco aislamiento térmico en sus casas, lo que los llevaba a percibir calor extremo dentro de sus casas en el verano y el frío en el invierno. En Hermosillo, esto tiene una explicación plausible; en el verano las temperaturas máximas a la sombra rebasan normalmente los cuarenta grados y pueden ocasionalmente acercarse a los cincuenta grados centígrados. Aunque el invierno no es tan riguroso, no es raro que las temperaturas bajen a menos de cero grados centígrados en la ciudad. Si esto se combina con el hecho de que las viviendas de las familias de escasos recursos no poseen aislamiento térmico, la sensación de calor y de frío que reportan las personas, especialmente las de extracción socioeconómica baja, está más que justificada.

La percepción de buena iluminación y poco ruido en las casas quizá obedezca, por un lado, al hecho de que la ciudad se encuentra en una de las zonas de mayor luminosidad del mundo, pero también a que resulta relativamente fácil adquirir y hacer funcionar focos y lámparas dentro de las viviendas durante la noche. Por otro lado, y a pesar del notable crecimiento experimentado por Hermosillo en las últimas tres décadas, la ciudad se encuentra aún lejos de experimentar la contaminación sónica de las grandes urbes mexicanas.

Los resultados más interesantes, sin embargo, se refirieron a la relación entre la habitabilidad $y$ el funcionamiento familiar. De acuerdo con la evidencia arrojada por el modelo estructural probado, a medida que se incrementan los niveles de una buena habitabilidad de la vivienda también se incrementan los patrones de convivencia familiar positiva. El tamaño de esta relación es saliente: un $25 \%$ de la variación en la convivencia es explicado por las condiciones de habitabilidad de la vivienda. Esto, aunado a los resultados de Corral et al., $(2010 ; 2011)$ señalaría que las condiciones físicas de la casa influyen en funcionamiento familiar, por lo que deberían ser tomadas en cuenta a la hora de evaluar y promover interacciones positivas entre los integrantes de una familia. Los componentes de la habitabilidad de la vivienda, por ejemplo, entrarían en la clasificación de indicadores de funcionamiento familiar (Zubrick et al., 2000), repartidos especialmente en las categorías de recursos materiales y de capital psicológico.

En este reporte consideramos a la habitabilidad como variable independiente y a la convivencia familiar como dependiente de la primera. Dado que nuestros datos no son experimentales (y por consideraciones de tipo ético no podrían serlo) es difícil asegurar con total certidumbre que el flujo causal se dé en la dirección planteada (de la habitabilidad a la convivencia). Es probable que esa dirección sea invertida, por lo menos en ciertos casos. Por ejemplo, en aquellas situaciones en las que un indicador de habitabilidad como el hacinamiento se veía afectado por la convivencia positiva, como lo discutimos previamente; quizá los bajos niveles de hacinamiento percibidos respondieran a que las interacciones familiares se percibieran más cercanas a los niveles óptimos y más alejados de los malos patrones de convivencia. La dirección causal convivencia familiar->habitabilidadtambién podríaplasmarse en la suposición de que las condiciones de habitabilidad sean una parte del funcionamiento familiar, por lo que en esa situación se consideraría que dicho funcionamiento afecta o promueve la habitabilidad. Una tercera opción es que habitabilidad y convivencia se afecten mutuamente, lo que, para el caso de las conclusiones y posibles recomendaciones vendría a significar que un cambio en condiciones de habitabilidad se vería necesariamente acompañado en modificaciones en los patrones de convivencia en la familia y que éstos, a su vez, afectarían las percepciones de habitabilidad desarrollados por los integrantes del grupo familiar.

También es necesario considerar que, en nuestro estudio, la habitabilidad fue influida 
por la antigüedad de la casa. Si el deterioro normal y la falta de mantenimiento a la casa se acumulan es muy probable que las condiciones físicas y también las psicológicas que constituyen la habitabilidad se vean afectadas. Dado que la antigüedad de la vivienda influye en su habitabilidad y ésta en la convivencia, lo anterior implica que el mantenimiento de la vivienda es otro elemento importante para promover no sólo habitabilidad, sino también una convivencia armónica en los hogares.

Por lo anteriormente expuesto, los datos, de ser replicados en investigaciones subsecuentes, sugerirían que a la hora de tomar decisiones en materia de políticas públicas relacionadas con la vivienda, las autoridades y planeadores deben considerar que las condiciones físicas de una casa no solo afectan el bienestar de un individuo, al proveerle salud física, refugio, satisfacción, identidad y un territorio para la apropiación (Aragonés \& Amérigo, 2010). Esas condiciones pueden afectar también la manera en la que se relacionan los integrantes de una familia, por lo que de su decisión de dotar a las viviendas con las condiciones apropiadas de habitabilidad dependería el que esa familia conviviera en un escenario propicio para la convivencia familiar positiva, y no en uno que genere estrés y malos tratos entre sus integrantes. 
Altman, I. (1975). The environment and social behavior. Monterey, CA: Brooks/Cole Publishers Company.

Amar, J. (1994). La educación infantil y desarrollo social. Barranquilla, Colombia: Ediciones Uninorte.

Aragonés, J.I. \& Amérigo, M. (2010). Psicología ambiental. Aspectos conceptuales y metodológicos. En J.I. Aragonés y M. Amérigo (coords.). Psicología Ambiental. Madrid: Editorial Pirámide.

Bentler, P.M. (2006). EQS, Structural Equations Program Manual. Encino, CA: Statistical Software, Inc.

Corral, V., Barrón, M., Cuén, A. y Tapia, C. (2011). Habitabilidad de la vivienda, estrés y violencia familiar. Psyecology, 2, 3-14.

Corral, V., Frías, M. \& González, D. (2010). Environmental factors in housing habitability as determinants of family violence. En M. Frías \& V. Corral (eds.). Biopsychosocial Perspectives on Interpersonal Violence. New York: Nova.

Harner, L. (1982). Talking about the past and the future. In, W. J. Friedman (ed.). The Development of the Psychology of Time. New York: Academic Press.

Herrenkohl, T. \& Herrenkohl, R. (2007). Examining the Overlap and Prediction of Multiple Forms of Child Maltreatment, Stressors, and Socioeconomic Status: A Longitudinal Analysis of Youth Outcomes. Journal of Family Violence, 22, 553-562.

Hillier, B. \& Hanson J. (1988). The social logic of space. Cambridge: Cambridge University Press.

Holman, E. \& Stokols (1994). The environmental psychology of child sexual abuse. The Journal of Environmental Psychology, $14,237-252$.

Hombrados, M.I. (2010). Hacinamiento. En J.I. Aragonés y M. Amérigo (coords.). Psicología Ambiental. Madrid: Editorial Pirámide.

Landázuri, A. \& Mercado, S. (2004). Algunos factores físicos y psicológicos relacionados con la habitabilidad interna de la vivienda. Medio Ambiente y Comportamiento Humano, 5, 89113.
Levi y Anderson L. (1980). La tensión psicosocial. PoblaciónAmbiente y calidad de vida. México: El manual moderno.

Mercado, S.J. (1998). La vivienda: Una perspectiva psicológica. En J. Guevara, A. M. Landázuri y A. Terán (coords.). Estudios de Psicología Ambiental en América Latina (pp. 141-153). México: BUAP-UNAM-CONACYT.

Montemayor, R. (1982). The relationship between parentadolescent conflict and the amount of time adolescents spend alone and with parents and peers. Child Development, 53, 1512-1519.

Noller, P., Seth-Smith, M., Bouma, R. \& Schweitzer, R. (1992). Parent and adolescent perceptions of family functioning: $A$ comparison of clinic and non-clinic families. Journal of family and Adolescence, 15, 101-114.

Piaget, J. (1969). The Child's Conception of Time. Londres: Routledge \& Kegan Paul.

Richardson, R.A., Abramowitz, R.H., Asp, C.E. \& Petersen, A.C. (1986). Parent-child relationships in early adolescence: Effects of family structure. Journal of Marriage and Family, 48, 805811.

Rueda, S. (2004). Habitabilidad y calidad de vida. Cuadernos de investigación Urbana, 42, 29-34.

Ruiz, M., Ropero, C., Amor, J. \& Amarís, M. (2003). Familia con violencia conyugal y su relación con la formación del autoconcepto. Psicología desde el Caribe, 11, 1-23.

Santoyo, C. \& Anguera, M.T. (1992). El hacinamiento como contexto: estrategias metodológicas para su análisis. Psicothema, 4, 551-569.

Valera, S. y Vidal, T. (2010). Privacidad y territorialidad. En J.I. Aragonés y M. Amérigo (coords.). Psicología Ambiental. Madrid: Editorial Pirámide.

Zubrick, S., Williams, A. \& Silburn, S. (2000). Indicators of social and family functioning. Canberra, Australia: Department of Family and Community Services. 